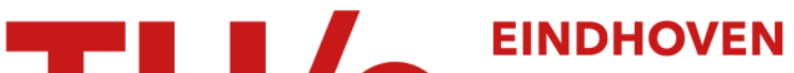

\section{Scattering hypervolume for ultracold bosons from weak to strong interactions}

\section{Citation for published version (APA):}

Mestrom, P. M. A., Colussi, V. E., Secker, T., \& Kokkelmans, S. J. J. M. F. (2019). Scattering hypervolume for ultracold bosons from weak to strong interactions. Physical Review A, 100(5), [050702].

https://doi.org/10.1103/PhysRevA.100.050702

DOI:

10.1103/PhysRevA.100.050702

Document status and date:

Published: 21/11/2019

\section{Document Version:}

Publisher's PDF, also known as Version of Record (includes final page, issue and volume numbers)

\section{Please check the document version of this publication:}

- A submitted manuscript is the version of the article upon submission and before peer-review. There can be important differences between the submitted version and the official published version of record. People interested in the research are advised to contact the author for the final version of the publication, or visit the $\mathrm{DOI}$ to the publisher's website.

- The final author version and the galley proof are versions of the publication after peer review.

- The final published version features the final layout of the paper including the volume, issue and page numbers.

Link to publication

\section{General rights}

Copyright and moral rights for the publications made accessible in the public portal are retained by the authors and/or other copyright owners and it is a condition of accessing publications that users recognise and abide by the legal requirements associated with these rights.

- Users may download and print one copy of any publication from the public portal for the purpose of private study or research.

- You may not further distribute the material or use it for any profit-making activity or commercial gain

- You may freely distribute the URL identifying the publication in the public portal.

If the publication is distributed under the terms of Article 25fa of the Dutch Copyright Act, indicated by the "Taverne" license above, please follow below link for the End User Agreement:

www.tue.nl/taverne

Take down policy

If you believe that this document breaches copyright please contact us at:

openaccess@tue.nl

providing details and we will investigate your claim. 
PHYSICAL REVIEW A 100, 050702(R) (2019)

Rapid Communications

\title{
Scattering hypervolume for ultracold bosons from weak to strong interactions
}

\author{
P. M. A. Mestrom $\odot,{ }^{*}$ V. E. Colussi, T. Secker, and S. J. J. M. F. Kokkelmans \\ Eindhoven University of Technology, P.O. Box 513, 5600 MB Eindhoven, The Netherlands
}

(Received 17 May 2019; published 21 November 2019)

\begin{abstract}
The elastic scattering properties of three bosons at low energy enter the many-body description of ultracold Bose gases via the three-body scattering hypervolume $D$. We study this quantity for identical bosons that interact via a pairwise finite-range potential. Our calculations cover the regime from strongly repulsive potentials towards attractive potentials supporting multiple two-body bound states and are consistent with the few existing predictions for $D$. We present a numerical confirmation of the universal predictions for $D$ in the strongly interacting regime, where Efimov physics dominates, for a local nonzero-range potential. Our findings highlight how $D$ is influenced by three-body quasibound states with strong $d$-wave or $g$-wave characteristics in the weakly interacting regime.
\end{abstract}

DOI: 10.1103/PhysRevA.100.050702

Introduction. Due to the precise experimental control of interatomic interactions via external magnetic fields, ultracold atomic gases have emerged as a versatile field for studying and manipulating quantum systems. The effective two-body interaction strength given by the $s$-wave scattering length $a$ can be tuned via Feshbach resonances [1]. When $|a|$ diverges, Efimov predicted the existence of an infinite number of three-body bound states whose universal scaling properties have been observed experimentally [2-8]. This nonperturbative threebody effect influences the properties of strongly interacting Bose gases [9-13] and Bose-Einstein condensates (BECs) interacting with an impurity particle [14-17]. Connecting few-body processes with bulk properties of ultracold Bose gases is fundamental to our understanding of these quantum many-body systems.

This connection is evident from a low-density expansion of the ground-state energy density $\mathcal{E}$ of a dilute BEC with a homogeneous number density $n[18]$ :

$$
\begin{aligned}
\mathcal{E}= & \frac{2 \pi \hbar^{2} n^{2} a}{m}\left\{1+\frac{128}{15 \sqrt{\pi}} \sqrt{n a^{3}}+\left[\frac{8(4 \pi-3 \sqrt{3})}{3} \ln \left(n a^{3}\right)\right.\right. \\
& \left.\left.+\frac{D}{12 \pi a^{4}}+\pi r_{s} / a+118.5\right] n a^{3}+\cdots\right\}
\end{aligned}
$$

where the dots indicate higher-order correction terms in the diluteness parameter $n a^{3}, m$ is the mass of a boson, $r_{s}$ is the two-body effective range, and $a>0$. The $\sqrt{n a^{3}}$ correction, calculated by Lee, Huang, and Yang (LHY) [19,20], originates from two-body elastic scattering characterized by $a$ alone. Experiments have probed LHY physics by measuring the critical temperature of a BEC [21], quantum depletion [22], excitation spectrum [23,24], thermodynamic equation of state [25], and contact [26]. Additionally, recent studies predicted [27] and experimentally confirmed the formation of quantum droplets in mixtures [28-30] and dipolar BECs [31-33] due to a stabilizing force originating from the LHY correction.

\footnotetext{
*Corresponding author: p.m.a.mestrom@tue.nl
}

As the study of strongly interacting Bose gases advances, there is the opportunity to observe beyond-LHY corrections. These corrections have been studied both phenomenologically, via extensions of the Gross-Pitaevskii equation [34-42], and microscopically, via quantum Monte Carlo simulations $[43,44]$ and studies of three-body scattering in vacuum [18,45-47]. Specifically, zero-energy three-body collisions determine the $\ln \left(n a^{3}\right)$ correction calculated by $\mathrm{Wu}$ [48-50] and the scattering hypervolume $D$ [18]. Crucially, $D$ determines the effective three-body interaction in an analogous role to $a$ in the two-body case. It is predicted to act as a stabilizing force for quantum droplets in ultracold Bose gases [38-40], may be tuned experimentally [51], and could be experimentally determined from the compressibility or sound modes of Bose gases [52].

The imaginary part of $D$ is proportional to the three-body recombination rate $[4,53]$ and has been studied extensively for various three-body systems, both experimentally and theoretically [54]. However, despite its fundamental relevance, the real part of $D$ remains sparsely explored. This is partly caused by the difficulty of removing singular contributions to the elastic three-body scattering amplitude required to obtain the real part of the scattering hypervolume $[4,6,45,55]$. In the strongly interacting regime, Efimov physics plays a dominant role leading to universal log-periodic behavior of $D$ $[4,6,47,56]$. In the weakly interacting regime, $D$ has been studied considering the repulsive hard-sphere potential [18] and a Gaussian interaction potential [53]. However, the behavior of $D$ over a full range of interaction strengths has not been explored for any finite-range potential, which demonstrates the nontrivial character of this problem.

In this Rapid Communication, we investigate the threebody scattering hypervolume $D$ for identical bosons interacting via a pairwise square-well potential, covering the range from weak to strong interactions, and analyze the corresponding universal and nonuniversal effects. We present numerical calculations of $D$ in the strongly interacting regime for a local finite-range potential, and study the corresponding Efimov universality. Besides the Efimov resonances, we identify 
additional three-body resonances close to two-body $d$ - and $g$-wave resonances and study their character.

Elastic three-body scattering amplitude. A convenient way to calculate $D$ is to use the Faddeev equations for the threeparticle transition operators $U_{\alpha \beta}$ in the form presented by Alt, Grassberger, and Sandhas (AGS) [57,58],

$$
\begin{aligned}
& U_{00}(z)=\sum_{\alpha=1}^{3} T_{\alpha}(z) G_{0}(z) U_{\alpha 0}(z), \\
& U_{\alpha 0}(z)=G_{0}^{-1}(z)+\sum_{\substack{\beta=1 \\
\beta \neq \alpha}}^{3} T_{\beta}(z) G_{0}(z) U_{\beta 0}(z)
\end{aligned}
$$

for $\alpha=1,2,3$,

to find the transition amplitude for three-body elastic scattering that is described by the operator $U_{00}(z)$. Here $z$ is the (complex) three-body energy. The index $\alpha(\beta)$ in $U_{\alpha \beta}(z)$ labels the four possible configurations for the outgoing (incoming) state of the three-body scattering wave function, i.e., $\alpha=0$ denotes three free particles, whereas $\alpha=1,2$, and 3 stand for the three possible atom-dimer configurations. $T_{\alpha}(z)$ represents the transition operator for scattering between particles $\beta$ and $\gamma(\beta, \gamma=1,2,3, \beta \neq \gamma \neq \alpha)$ in the presence of particle $\alpha$ and is simply related to the two-body $T$ operator $T\left(z_{2 b}\right)$ [59], where $z_{2 b}$ is some complex value for the energy of the two-body system. The operator $G_{0}(z)$ is the free threebody Green's function $\left(z-H_{0}\right)^{-1}$ where $H_{0}$ is the three-body kinetic energy operator in the center-of-mass frame of the three-particle system.

The three-body configuration is parametrized by the Jacobi momenta $\mathbf{p}_{\alpha}=\left(\mathbf{P}_{\beta}-\mathbf{P}_{\gamma}\right) / 2$ and $\mathbf{q}_{\alpha}=$ $(2 / 3)\left[\mathbf{P}_{\alpha}-\left(\mathbf{P}_{\beta}+\mathbf{P}_{\gamma}\right) / 2\right]$ where $\mathbf{P}_{\alpha}$ represents the momentum of particle $\alpha$ in the laboratory frame. There exist three possibilities to choose these Jacobi vectors. If we define $\mathbf{q} \equiv \mathbf{q}_{1}$ and $\mathbf{p} \equiv \mathbf{p}_{1}$, we have $\mathbf{q}_{2}=\mathbf{p}-\mathbf{q} / 2, \quad \mathbf{p}_{2}=-\mathbf{p} / 2-3 \mathbf{q} / 4, \quad \mathbf{q}_{3}=-\mathbf{p}-\mathbf{q} / 2, \quad$ and $\mathbf{p}_{3}=-\mathbf{p} / 2+3 \mathbf{q} / 4$. This parametrization is suitable for relating the matrix element $\left\langle\mathbf{p}, \mathbf{q}\left|U_{00}(0)\right| \mathbf{0}, \mathbf{0}\right\rangle$ to the scattering hypervolume $D$ where we normalize the plane-wave states according to $\left\langle\mathbf{p}^{\prime} \mid \mathbf{p}\right\rangle=\delta\left(\mathbf{p}^{\prime}-\mathbf{p}\right)$. From Tan's definition of the three-body scattering hypervolume $D$ [18], we deduce the following relation between $\left\langle\mathbf{p}, \mathbf{q}\left|U_{00}(0)\right| \mathbf{0}, \mathbf{0}\right\rangle$ and $D$ (see Supplemental Material [60]):

$$
\begin{aligned}
\left\langle\mathbf{p}, \mathbf{q}\left|U_{00}(0)\right| \mathbf{0}, \mathbf{0}\right\rangle= & \sum_{\alpha=1}^{3}\left\{\delta\left(\mathbf{q}_{\alpha}\right)\left\langle\mathbf{p}_{\alpha}|T(0)| \mathbf{0}\right\rangle-\frac{1}{2 \pi^{4}} \frac{a^{2}}{m \hbar^{2}} \frac{1}{q_{\alpha}^{2}}+\frac{1}{12 \pi^{4}}(4 \pi-3 \sqrt{3}) \frac{a^{3}}{m \hbar^{3}} \frac{1}{q_{\alpha}}+\frac{1}{3 \pi^{5}}(4 \pi-3 \sqrt{3}) \frac{a^{4}}{m \hbar^{4}} \ln \left(\frac{q_{\alpha}|a|}{\hbar}\right)\right. \\
& \left.-\left.\frac{p_{\alpha}^{2}+\frac{3}{4} q_{\alpha}^{2}}{q_{\alpha}^{2}} \frac{a}{2 \pi^{2} \hbar} \frac{\partial^{2}\langle\mathbf{p}|T(0)| \mathbf{0}\rangle}{\partial p^{2}}\right|_{p=0}+\frac{1}{3} \frac{1}{(2 \pi)^{6}} \frac{D}{m \hbar^{4}}+O\left[q_{\alpha} \ln \left(\frac{q_{\alpha}|a|}{\hbar}\right), \frac{p_{\alpha}^{2}}{q_{\alpha}}\right]\right\}
\end{aligned}
$$

which holds for any local symmetric two-body potential. Here the three-body energy $z=0$ is approached from the upper half of the complex energy plane, which fixes the sign of the imaginary part of $D$.

We consider three identical bosons that interact via a pairwise square-well potential

$$
V(r)= \begin{cases}-V_{0}, & 0 \leqslant r<R, \\ 0, & r \geqslant R,\end{cases}
$$

where $r$ denotes the relative distance between two particles and $R$ and $V_{0}$ represent the range and depth of the potential, respectively. To obtain $D$, we solve the AGS equations given in Eq. (2) for the matrix element $\left\langle\mathbf{p}, \mathbf{q}\left|U_{00}(0)\right| \mathbf{0}, \mathbf{0}\right\rangle$ after subtracting the terms in Eq. (3) that diverge as $p, q \rightarrow 0$ (see Ref. [60]). The dimension of this set of integral equations is reduced to one by expanding this amplitude in spherical harmonics and in two-body states that are determined by the Weinberg expansion and are thus related to two-body bound states or resonances $[59,61]$. The resulting integral equation is solved as a matrix equation by discretizing the momenta.

Our method differs from another approach recently presented by Zhu and Tan [53] who calculated the scattering hypervolume $D$ from the zero-energy three-body scattering wave function in position space for a variable two-body Gaussian potential. Their numerics were limited to the weakly interacting regime in contrast to our approach covering the complete regime ranging from strongly repulsive to attractive potentials and from weak $(|a| / R \lesssim 1)$ to strong $(|a| / R \gg 1)$ interactions. In the following, we show our results in these regimes obtained by tuning the potential depth $V_{0}$.

Repulsive potentials. In the limit $V_{0} \rightarrow-\infty$, the squarewell potential approaches the hard-sphere interaction that was considered already a decade ago by Tan [18]. Our results for $D$ in this limit are shown in Fig. 1(a) where we find good agreement within the numerical accuracy of our approach:

$$
D / a^{4} \underset{\bar{V}_{0} \rightarrow-\infty}{=} 1761 \pm 1 .
$$

When the potential barrier $-V_{0}$ is decreased, the scattering hypervolume decreases as well, and it eventually goes to zero in the limit $\left|V_{0}\right| \rightarrow 0$ as

$$
\begin{aligned}
D & =-\left.96 \pi^{6} m^{2} \hbar^{4}\langle\mathbf{0}|V| \mathbf{0}\rangle \frac{\partial^{2}\langle\mathbf{p}|V| \mathbf{0}\rangle}{\partial p^{2}}\right|_{p=0}+O\left(\bar{V}_{0}^{3}\right) \\
& =\frac{8}{15} \pi^{2} \bar{V}_{0}^{2} R^{4}+O\left(\bar{V}_{0}^{3}\right),
\end{aligned}
$$

where $\bar{V}_{0}=m V_{0} R^{2} / \hbar^{2}$ denotes the dimensionless interaction strength. Equation (6) is a general relation for $D$ for local symmetric potentials $V$ in the zero-depth limit [62], whereas Eq. (7) applies specifically to the square-well potential. We have analytically derived Eq. (6) from the AGS equations using the Born approximation (see Ref. [60]), and we have numerically confirmed it for the square-well potential. An 


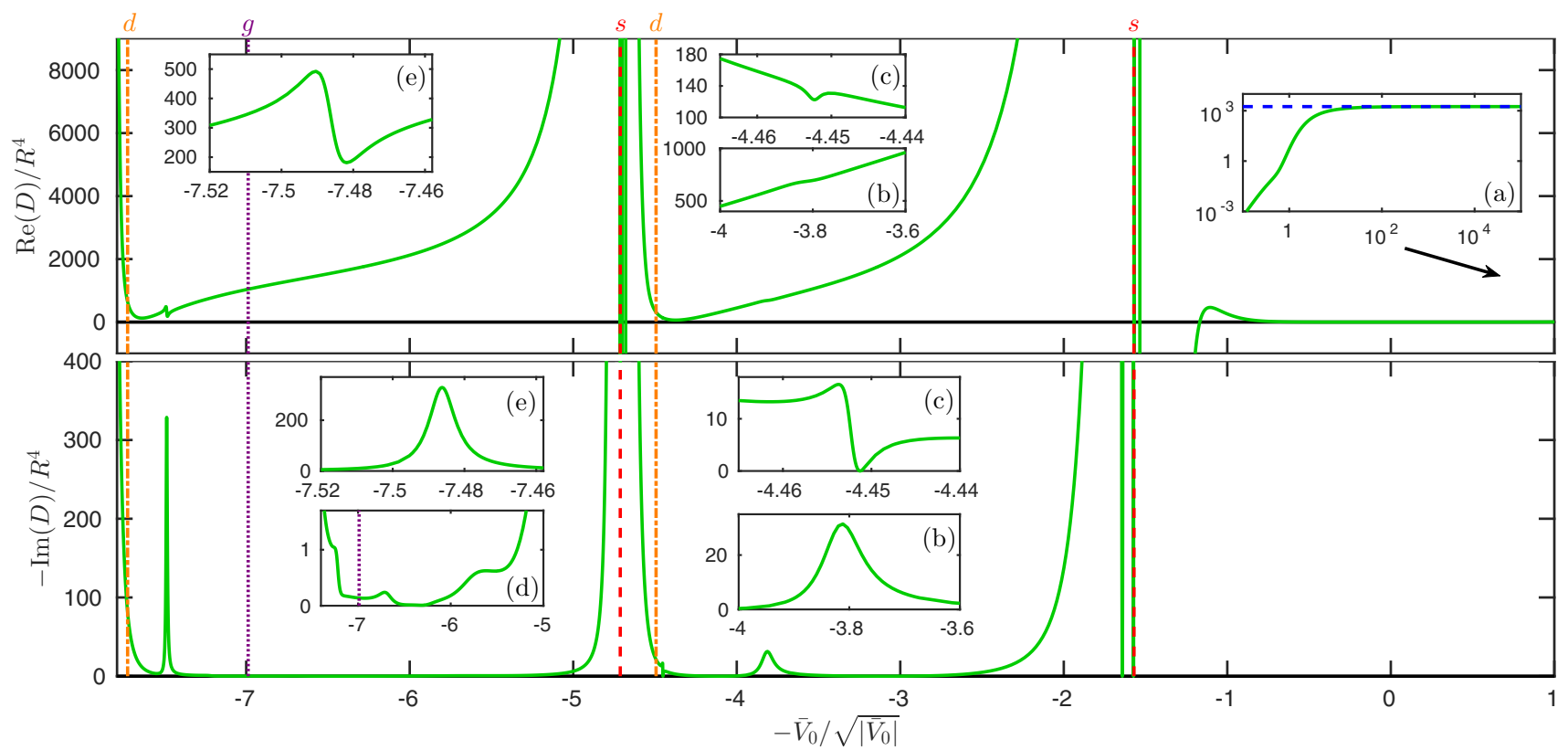

FIG. 1. Three-body scattering hypervolume $D$ (green solid line) corresponding to the square-well potential as a function of the dimensionless interaction strength $\bar{V}_{0}=m V_{0} R^{2} / \hbar^{2}$. The vertical lines indicate the interaction strengths at which two-body states become bound: $s$-wave states $\left(l=0\right.$, red dashed lines) at $\bar{V}_{0}=(\pi / 2)^{2}$ and $(3 \pi / 2)^{2}, d$-wave states $\left(l=2\right.$, orange dash-dotted lines) at $\bar{V}_{0} \approx(4.49)^{2}$ and $(7.73)^{2}$, and $g$-wave state $\left(l=4\right.$, purple dotted line) at $\bar{V}_{0} \approx(6.99)^{2}$. Inset (a) displays the behavior of $D$ for strongly repulsive potentials as indicated by the black arrow. The horizontal blue dashed line represents the hard-sphere limit calculated by Ref. [18]. The other insets (b)-(e) zoom in on the real and imaginary parts of $D$ near several resonances that arise from three-body quasibound states at the three-particle threshold.

expression equivalent to Eq. (6) has been derived in position space by Ref. [53] that confirmed it for a Gaussian potential.

Attractive potentials. As the potential depth increases, twobody states start to become bound resulting in a nonzero value for the imaginary part of $D$. Figure 1 shows that this value is much smaller than the magnitude of the real part in most regimes. Close to the two-body $s$-wave potential resonances that are indicated by the vertical red dashed lines in Fig. 1, the pairwise interactions are strong $(|a| \gg R)$. Here the scattering hypervolume $D$ scales as $a^{4}$ and its behavior becomes log-periodic due to the Efimov effect as we will see below. In between these two-body resonances where $|a| \lesssim R$, we identify several resonances related to three-body quasibound states that appear at the zero-energy threshold as indicated by Figs. 1(b)-1(e). In the following paragraphs, we first analyze the characteristics of these three-body resonances before presenting our results in the strongly interacting regime.

The presence of the trimer resonances at $\sqrt{\bar{V}_{0}}=3.8$ and 4.45 [Figs. 1(b) and 1(c), respectively] depends critically on the inclusion of the almost bound two-body $d$-wave state (vertical orange dash-dotted line at $\sqrt{\bar{V}_{0}} \approx 4.49$ ) in our Weinberg expansion of the two-body $T$ operator. This suggests that these trimer states are associated with this $d$-wave dimer state in a similar way as the three-body state for van der Waals potentials studied by Ref. [63].

In Fig. 1(d) we highlight small features at $\sqrt{\bar{V}_{0}}=5.7$, 6.7, and 7.2. These are close to the point at which the first $g$-wave dimer state gets bound (vertical purple dotted line).
By analyzing the eigenvalues of the kernel of the integral equation (see Ref. [60]), we find that they are true trimer resonances. These resonances vanish when the first $g$-wave dimer state is removed from the Weinberg expansion. We do not see any effects of the resonances at $\sqrt{\bar{V}_{0}}=5.7,6.7$, and 7.2 on the real part of $D$ within our numerical accuracy. More generally, our results suggest that trimer resonances in the weakly interacting regime have a stronger effect on $\operatorname{Im}(D)$ than on $\operatorname{Re}(D)$.

The next trimer resonance occurs at $\sqrt{\bar{V}_{0}}=7.49$ [see Fig. 1(e)]. It vanishes when removing the second $d$-wave or the second $g$-wave dimer state from our Weinberg expansion. These two-body states get bound at $\sqrt{\bar{V}_{0}} \approx 7.73$ (vertical orange dash-dotted line) and $\sqrt{\bar{V}_{0}} \approx 10.42$, respectively. So both $d$-wave and $g$-wave effects play a significant role for this trimer resonance.

Even though these trimer resonances in the weakly interacting regime all originate from the nonzero partial-wave components of the two-body interaction potential, the behavior of $D$ is not the same for all resonances. This suggests that the behavior of the scattering hypervolume in the weakly interacting regime depends on some three-body background phase shift resulting from nonresonant pathways for threebody scattering [6]. In particular, Fig. 1(c) shows a sharp minimum in $-\operatorname{Im}(D)$ (or equivalently in the three-body recombination rate) near the trimer resonance peak at $\sqrt{\bar{V}_{0}}=4.45$. Such a sharp feature was also encountered by Ref. [53] for a Gaussian potential supporting two $s$-wave dimer states. We 
suspect that both features arise from destructive interference effects [6], since the minimum in Fig. 1(c) vanishes when we exclude the almost bound two-body $d$-wave state in our Weinberg expansion.

Our results presented in Fig. 1 can be compared to the calculations of Ref. [53] for the scattering hypervolume corresponding to a Gaussian two-body potential. Even though both results are very similar for repulsive potentials, they are quite different for attractive interactions. The main difference is the behavior of $D$ when approaching the $s$-wave dimer resonances (vertical red dashed lines), where Ref. [53] finds additional trimer resonances that are different from the Efimov resonances. Secondly, we find that $D$ behaves smoothly across the $d$-wave dimer resonances (vertical orange dash-dotted lines) in contrast to the results of Ref. [53]. These differences show that the details of the considered two-body potential play a crucial role in the behavior of $D$ across a $d$-wave dimer resonance and on the presence of trimer states in the weakly interacting regime.

We now discuss our results in the strongly interacting regime $(|a| / R \gg 1)$. Here, the behavior of $D$ is predicted to follow a general form determined in Refs. [47,56,64-66] and generalized in Refs. $[4,6,67]$ by including the inelasticity parameter $\eta$ that describes the tendency to decay to deeply bound dimer states. These limiting forms for $D$ contain a number of universal constants obtained in Refs. [47,67-70] which we refine in this work (see Ref. [60]). In addition to $\eta$, they also depend on the nonuniversal parameters $a_{-}$and $a_{+}$ that locate the three-body recombination maxima and minima, respectively, and are completely determined by the interaction between the three particles [4].

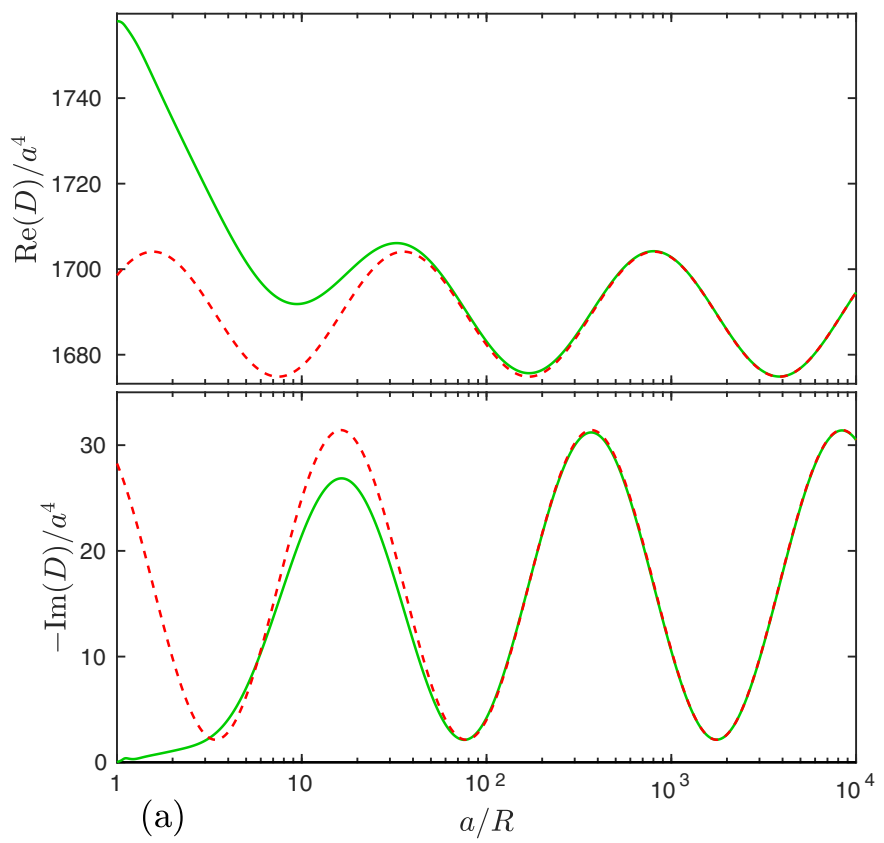

The universal expressions for the real part of the scattering hypervolume $D$ are given by

$$
\operatorname{Re}\left(D / a^{4}\right) \approx C\left(c_{-}+\frac{\frac{1}{2} b_{-} \sin \left[2 s_{0} \ln \left(a / a_{-}\right)\right]}{\sin ^{2}\left[s_{0} \ln \left(a / a_{-}\right)\right]+\sinh ^{2}(\eta)}\right)
$$

for $a<0$ and

$$
\begin{aligned}
\operatorname{Re}\left(D / a^{4}\right) \approx & C\left(c_{+}+\frac{1}{2} b_{+}\left(1-e^{-2 \eta}\right)\right. \\
& \left.+b_{+} e^{-2 \eta} \sin ^{2}\left[s_{0} \ln \left(a / a_{+}\right)-\pi / 4\right]\right)
\end{aligned}
$$

for $a>0$. The imaginary part of $D$ is given by the universal formulas

$$
\operatorname{Im}\left(D / a^{4}\right) \approx-\frac{1}{2} C_{-} \frac{\sinh (2 \eta)}{\sin ^{2}\left[s_{0} \ln \left(a / a_{-}\right)\right]+\sinh ^{2}(\eta)}
$$

for $a<0$ and

$$
\begin{aligned}
\operatorname{Im}\left(D / a^{4}\right) \approx & -\frac{1}{2} C_{+}\left(\frac{1}{4}\left(1-e^{-4 \eta}\right)\right. \\
& \left.+e^{-2 \eta}\left\{\sin ^{2}\left[s_{0} \ln \left(a / a_{+}\right)\right]+\sinh ^{2}(\eta)\right\}\right)
\end{aligned}
$$

for $a>0$. Here $s_{0} \approx 1.00624$ is the constant that sets the periodicity in Efimov physics for identical bosons [2,3] and we have defined the constant $C \equiv 64 \pi(4 \pi-3 \sqrt{3})$. The coefficients $b_{ \pm}, c_{ \pm}$, and $C_{ \pm}$are universal in the sense that they do not depend on the short-range form of the potentials [6]. These constants were determined previously to be $C_{-} \approx 4590$ [67], $C_{+} \approx 67.1177$ [68-70], $b_{-}=3.16, c_{-}=1.14, b_{+}=0.021$, and $c_{+}=1.13$ [47] (see Ref. [60] for the connection between $D$ and the quantity calculated in Ref. [47]).

We have redetermined the universal coefficients as $C_{+}=$ 67.118(5), $b_{+}=0.0226(5), c_{+}=1.1288(5), b_{-}=3.153(5)$,

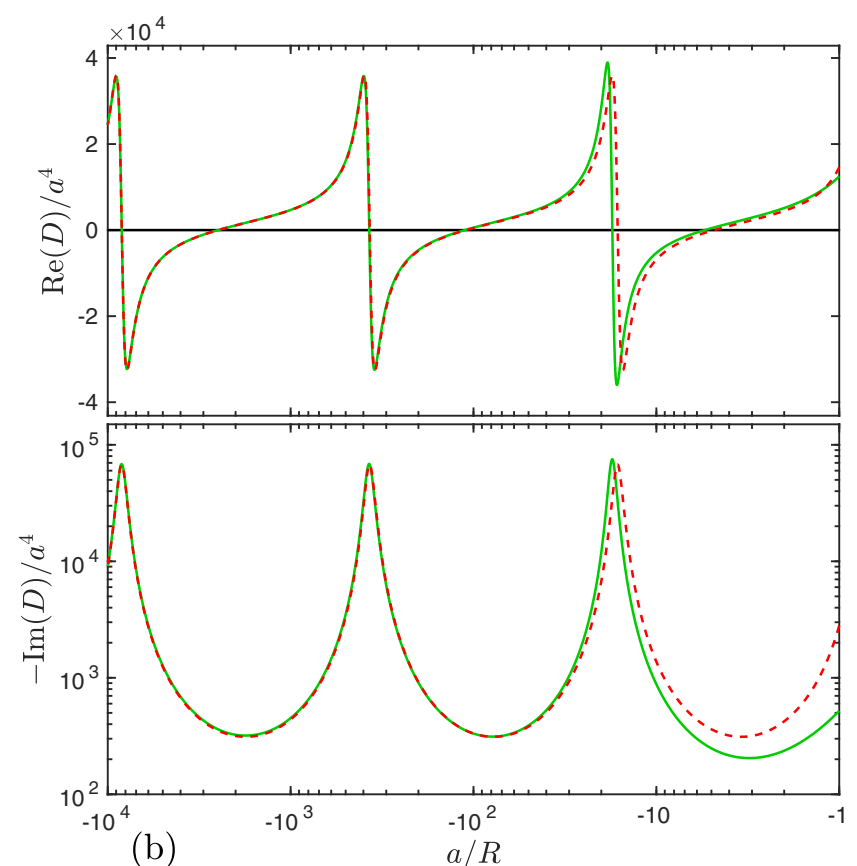

(b)

FIG. 2. Three-body scattering hypervolume $D$ (green solid line) near the second potential resonance of the square-well potential for (a) $a>$ 0 and (b) $a<0$. The dashed curves give the analytic zero-range results given by Eqs. (8), (9), (10), and (11) where we set $a_{+} / R=1759$, $b_{+}=0.0226, c_{+}=1.1288, C_{+}=67.118, a_{-} / R=-8396, b_{-}=3.153, c_{-}=1.140, C_{-}=4590$, and $\eta=0.068$. 
and $c_{-}=1.140(2)$. This was done by analyzing the threebody scattering hypervolume for a contact interaction with a cutoff in momentum space (see Ref. [60] for details). We find good agreement with the previously determined values except for $b_{+}$that deviates approximately $7 \%$ from Ref. [47]. However, this leads only to a deviation of $0.1 \%$ in the overall value of $D$ (see Ref. [60]).

The universal relations (8) and (9) have not been previously tested numerically for any local nonzero-range two-body potential. Near the second two-body $s$-wave potential resonance of the square-well potential, i.e., $\sqrt{\bar{V}_{0}}$ is close to $3 \pi / 2$, we compare our results against the universal relations in Fig. 2. Using our results for the universal constants, we numerically confirm Eqs. (8), (9), (10), and (11). For this specific two-body resonance, we find that $a_{+} / R=1759(5)$, $a_{-} / R=-8396(1)$, and $\eta=0.068(1)$. Similar results for the first potential resonance can be found in the Supplemental Material [60].

Conclusion. By solving the AGS equations for the threebody elastic scattering amplitude, we have studied the behavior of the three-body scattering hypervolume $D$ which is a fundamental quantity of ultracold three-body collisions and is needed for studying ultracold Bose gases beyond the LHY correction. We have presented numerical calculations of $D$ for identical bosons with a variable nonzero-range potential in the strongly interacting regime. Our results agree with the universal predictions of Refs. [4,6,47,56,64-67] and show how finite-range effects start to play a role as the absolute value of the scattering length is decreased. For repulsive interactions, we have confirmed the hard-sphere limit from Ref. [18] and the weak-interaction limit from Ref [53]. We have also explored the weakly interacting regime for attractive potentials supporting up to two $s$-wave dimer states and identified several three-body resonances related to trimer states that depend strongly on $d$-wave and/or $g$-wave effects.

The approach outlined in this Rapid Communication is very general and can be applied to other types of two-body potentials as well, such as van der Waals potentials. It could also be applied to mixtures, for which low-energy elastic three-body scattering properties are completely unexplored. Additionally, one could extend our approach to study threebody scattering embedded in a many-body environment [9] and determine how three-body correlations affect both stationary and dynamical observables of ultracold Bose gases for any short-range two-body potential. In particular, one could make quantitative predictions for the ground-state energy density of a BEC and investigate stabilizing effects from the three-body scattering hypervolume for small negative scattering lengths including the formation of quantum droplets [38-40].

Acknowledgments. We thank José P. D'Incao, Chris H. Greene, Silvia Musolino, Denise Braun, Gijs Groeneveld, and Jinglun Li for useful discussions. This research is financially supported by the Netherlands Organisation for Scientific Research (NWO) under Grant No. 680-47-623, and by the Foundation for Fundamental Research on Matter (FOM).
[1] C. Chin, R. Grimm, P. Julienne, and E. Tiesinga, Rev. Mod. Phys. 82, 1225 (2010).

[2] V. Efimov, Phys. Lett. B 33, 563 (1970).

[3] V. Efimov, Yad. Fiz. 12, 1080 (1970) [Sov. J. Nucl. Phys. 12, 589 (1971)].

[4] E. Braaten and H.-W. Hammer, Phys. Rep. 428, 259 (2006).

[5] P. Naidon and S. Endo, Rep. Prog. Phys. 80, 056001 (2017).

[6] J. P. D’Incao, J. Phys. B: At., Mol. Opt. Phys. 51, 043001 (2018).

[7] T. Kraemer, M. Mark, P. Waldburger, J. Danzl, C. Chin, B. Engeser, A. Lange, K. Pilch, A. Jaakkola, H.-C. Nägerl et al., Nature (London) 440, 315 (2006).

[8] B. Huang, L. A. Sidorenkov, R. Grimm, and J. M. Hutson, Phys. Rev. Lett. 112, 190401 (2014).

[9] V. E. Colussi, S. Musolino, and S. J. J. M. F. Kokkelmans, Phys. Rev. A 98, 051601(R) (2018).

[10] E. Braaten, D. Kang, and L. Platter, Phys. Rev. Lett. 106, 153005 (2011).

[11] F. Werner and Y. Castin, Phys. Rev. A 86, 053633 (2012).

[12] V. E. Colussi, J. P. Corson, and J. P. D’Incao, Phys. Rev. Lett. 120, 100401 (2018).

[13] V. E. Colussi, B. E. van Zwol, J. P. D’Incao, and S. J. J. M. F. Kokkelmans, Phys. Rev. A 99, 043604 (2019).

[14] J. Levinsen, M. M. Parish, and G. M. Bruun, Phys. Rev. Lett. 115, 125302 (2015)

[15] S. M. Yoshida, S. Endo, J. Levinsen, and M. M. Parish, Phys. Rev. X 8, 011024 (2018).

[16] M. Sun, H. Zhai, and X. Cui, Phys. Rev. Lett. 119, 013401 (2017).
[17] P. Naidon, J. Phys. Soc. Jpn. 87, 043002 (2018).

[18] S. Tan, Phys. Rev. A 78, 013636 (2008).

[19] T. D. Lee and C. N. Yang, Phys. Rev. 105, 1119 (1957).

[20] T. D. Lee, K. Huang, and C. N. Yang, Phys. Rev. 106, 1135 (1957).

[21] R. P. Smith, R. L. D. Campbell, N. Tammuz, and Z. Hadzibabic, Phys. Rev. Lett. 106, 250403 (2011).

[22] R. Lopes, C. Eigen, N. Navon, D. Clément, R. P. Smith, and Z. Hadzibabic, Phys. Rev. Lett. 119, 190404 (2017).

[23] S. B. Papp, J. M. Pino, R. J. Wild, S. Ronen, C. E. Wieman, D. S. Jin, and E. A. Cornell, Phys. Rev. Lett. 101, 135301 (2008).

[24] R. Lopes, C. Eigen, A. Barker, K. G. H. Viebahn, M. Robert-deSaint-Vincent, N. Navon, Z. Hadzibabic, and R. P. Smith, Phys. Rev. Lett. 118, 210401 (2017).

[25] N. Navon, S. Piatecki, K. Günter, B. Rem, T. C. Nguyen, F. Chevy, W. Krauth, and C. Salomon, Phys. Rev. Lett. 107, 135301 (2011).

[26] R. J. Wild, P. Makotyn, J. M. Pino, E. A. Cornell, and D. S. Jin, Phys. Rev. Lett. 108, 145305 (2012).

[27] D. S. Petrov, Phys. Rev. Lett. 115, 155302 (2015).

[28] C. R. Cabrera, L. Tanzi, J. Sanz, B. Naylor, P. Thomas, and L. Cheiney, P. Tarruell, Science 359, 301 (2018).

[29] P. Cheiney, C. R. Cabrera, J. Sanz, B. Naylor, L. Tanzi, and L. Tarruell, Phys. Rev. Lett. 120, 135301 (2018).

[30] G. Semeghini, G. Ferioli, L. Masi, C. Mazzinghi, L. Wolswijk, F. Minardi, M. Modugno, G. Modugno, M. Inguscio, and M. Fattori, Phys. Rev. Lett. 120, 235301 (2018).

[31] H. Kadau, M. Schmitt, M. Wenzel, C. Wink, T. Maier, I. FerrierBarbut, and T. Pfau, Nature (London) 530, 194 (2016). 
[32] I. Ferrier-Barbut, H. Kadau, M. Schmitt, M. Wenzel, and T. Pfau, Phys. Rev. Lett. 116, 215301 (2016).

[33] L. Chomaz, S. Baier, D. Petter, M. J. Mark, F. Wächtler, L. Santos, and F. Ferlaino, Phys. Rev. X 6, 041039 (2016).

[34] N. Akhmediev, M. P. Das, and A. V. Vagov, Int. J. Mod. Phys. B 13, 625 (1999).

[35] A. Gammal, T. Frederico, L. Tomio, and P. Chomaz, J. Phys. B: At., Mol. Opt. Phys. 33, 4053 (2000).

[36] A. Gammal, T. Frederico, L. Tomio, and P. Chomaz, Phys. Rev. A 61, 051602(R) (2000).

[37] S. K. Adhikari, Phys. Rev. A 66, 013611 (2002).

[38] A. Bulgac, Phys. Rev. Lett. 89, 050402 (2002).

[39] P. F. Bedaque, A. Bulgac, and G. Rupak, Phys. Rev. A 68, 033606 (2003).

[40] P. B. Blakie, Phys. Rev. A 93, 033644 (2016).

[41] H. Al-Jibbouri, I. Vidanović, A. Balaž, and A. Pelster, J. Phys. B: At., Mol. Opt. Phys. 46, 065303 (2013).

[42] K.-T. Xi and H. Saito, Phys. Rev. A 93, 011604(R) (2016).

[43] S. Giorgini, J. Boronat, and J. Casulleras, Phys. Rev. A 60, 5129 (1999).

[44] M. Rossi, L. Salasnich, F. Ancilotto, and F. Toigo, Phys. Rev. A 89, 041602(R) (2014)

[45] E. Braaten and A. Nieto, Eur. Phys. J. B 11, 143 (1999).

[46] T. Köhler, Phys. Rev. Lett. 89, 210404 (2002).

[47] E. Braaten, H.-W. Hammer, and T. Mehen, Phys. Rev. Lett. 88, 040401 (2002)

[48] T. T. Wu, Phys. Rev. 115, 1390 (1959).

[49] K. Sawada, Phys. Rev. 116, 1344 (1959).

[50] N. M. Hugenholtz and D. Pines, Phys. Rev. 116, 489 (1959).

[51] D. S. Petrov, Phys. Rev. Lett. 112, 103201 (2014).

[52] W. Zwerger, J. Stat. Mech.: Theory Expt. (2019) 103104.

[53] S. Zhu and S. Tan, arXiv:1710.04147v1.

[54] C. H. Greene, P. Giannakeas, and J. Pérez-Ríos, Rev. Mod. Phys. 89, 035006 (2017).
[55] R. D. Amado and M. H. Rubin, Phys. Rev. Lett. 25, 194 (1970).

[56] V. Efimov, Sov. J. Nucl. Phys. 29, 546 (1979).

[57] E. Alt, P. Grassberger, and W. Sandhas, Nucl. Phys. B 2, 167 (1967).

[58] E. W. Schmid and H. Ziegelmann, The Quantum Mechanical Three-Body Problem (Pergamon, Oxford, 1974).

[59] P. M. A. Mestrom, T. Secker, R. M. Kroeze, and S. J. J. M. F. Kokkelmans, Phys. Rev. A 99, 012702 (2019).

[60] See Supplemental Material at http://link.aps.org/supplemental/ 10.1103/PhysRevA.100.050702 for additional details of our calculations, derivations and results, which includes Ref. [71].

[61] S. Weinberg, Phys. Rev. 131, 440 (1963).

[62] For this general case, $\bar{V}_{0}$ is defined to be a prefactor of the potential as $V(r)=\bar{V}_{0} f(r)$ where $f(r)$ is independent of $\bar{V}_{0}$.

[63] J. Wang, J. P. D'Incao, Y. Wang, and C. H. Greene, Phys. Rev. A 86, 062511 (2012)

[64] B. D. Esry, C. H. Greene, and J. P. Burke, Phys. Rev. Lett. 83, 1751 (1999).

[65] P. F. Bedaque, E. Braaten, and H.-W. Hammer, Phys. Rev. Lett. 85, 908 (2000).

[66] E. Braaten and H.-W. Hammer, Phys. Rev. Lett. 87, 160407 (2001).

[67] E. Braaten and H.-W. Hammer, Phys. Rev. A 70, 042706 (2004).

[68] D. S. Petrov, Three-boson problem near a narrow Feshbach resonance, talk at the Workshop on Strongly Interacting Quantum Gases, Ohio State University, 2005.

[69] J. H. Macek, S. Y. Ovchinnikov, and G. Gasaneo, Phys. Rev. A 73, 032704 (2006).

[70] A. O. Gogolin, C. Mora, and R. Egger, Phys. Rev. Lett. 100, 140404 (2008).

[71] J. R. Taylor, Scattering Theory: The Quantum Theory on Nonrelativistic Collisions (Wiley, New York, 1972). 